\title{
Early protein-restriction-induced hyperphagia: a behavioural analysis
}

\author{
R. Orozco-Solis ${ }^{1}$, S. Lopes De Souza ${ }^{1,2}$, R. Manhaes de Castro ${ }^{2}$ and F. Bolaños-Jimenez ${ }^{1}$ \\ ${ }^{1}$ INRA, Nantes France and ${ }^{2}$ Universidade Federal de Pernambuco Pernambuco Brazil
}

Experimental studies in animals have shown that protein or energy restriction during gestation and/or suckling induces hyperphagia, a preference for high-fat food and the development in the long term of obesity, glucose intolerance and hypertension ${ }^{(1,2)}$. The offspring of dams fed a low-protein diet during gestation also exhibit reduced numbers of galanin and neuropeptide Y (NPY) neurons in the arcuate hypothalamic nucleus ${ }^{(3)}$ as well as an alteration in the levels of expression of several peptide neurotransmitters that modulate feeding such as NPY and cholecystokinin ${ }^{(4,5)}$. These observations have led to the proposal that a dysfunction of the neuronal circuits regulating food intake is a key step in the development of obesity associated with malnutrition in early life. To date, all the behavioural studies on the developmental programming of feeding have relied exclusively on the measurement of the amount of food consumed over a $12 \mathrm{~h}$ or a $24 \mathrm{~h}$ period. However, measurement of food intake alone does not enable the differentiation of specific and non-specific effects on feeding and does not provide any information about the underlying mechanism by which early nutrient restriction affects the normal physiological regulation of appetite, i.e. through changes in hunger motivation and/or satiety. The aim of the present study was to identify intrinsic differences in feeding patterns between control and perinatally-undernourished rats.

The temporal pattern of feeding and the behavioural satiety sequence under conditions of standard chow intake were investigated in the young offspring of Sprague-Dawley rats fed either a control $(200 \mathrm{~g} / \mathrm{kg})$ or a low-protein $(80 \mathrm{~g} / \mathrm{kg}$; LP) diet throughout pregnancy and lactation. The feeding pattern and some metabolic variables were examined in control and LP rats at $240 \mathrm{~d}$ of age under conditions of exposure to a high-fat $(260 \mathrm{~g} / \mathrm{kg})$ diet in order to determine whether the hyperphagia observed in the offspring of malnourished animals plays a role in determining the development of obesity and the maintenance of the obese state once established.

In agreement with previous observations ${ }^{(6-8)}$, during the first weeks after weaning LP pups consumed more food than controls. This hyperphagia was evident only when the amount of ingested food was normalised for body weight, since in absolute terms rats exposed to an LP diet during gestation and lactation ingested less food than control animals. During a single test meal both control and LP animals exhibited a typical behavioural satiety sequence characterised by the steady progression from an initial phase of eating through active and grooming behaviour to a phase of resting. However, LP animals spent significantly more time (min) feeding than controls (19.8 (SE 0.74 ) v. 17.0 (SE 0.86); $P<0.05$ ), which was accompanied by an increase in meal size (kJ/100 g body weight; 40.2 (SEM 2.9$)$ v. 25.0 (SE 2.34 ); $P<0.01)$ and a delay in the transition from eating to resting. LP pups also exhibited reduced latency to eat in relation to well-nourished pups (27.1 (SE 5.04) s $v$. 46.1 (SE 5.15) s; $P<0.05$ ). In contrast, no significant differences in feeding rate were observed between the two groups. From $60 \mathrm{~d}$ onwards no significant differences in spontaneous food intake of standard chow were evident between control and perinatally-undernourished animals. Unexpectedly, however, under conditions of exposure to a high-fat diet 240-d-old control animals consumed more food than LP rats. The inspection of the circadian feeding behaviour indicated that this increased feeding took place during the light phase of the dark-light cycle. On the standard chow the old LP rats already exhibited clear disturbances characteristic of obese rats, including elevated plasma leptin and TAG along with increased visceral fat. Plasma cholesterol and fatty acids were also increased after ingestion of the high-fat diet.

Altogether, these observations suggest that susceptibility to perinatal undernutrition-induced obesity is independent of the developmental programming of feeding.

1. Remacle C, Bieswal F \& Reusens B (2004) Int J Obes Relat Metab Disord 28, S46-S53.

2. Armitage JA, Khan IY, Taylor PD et al. (2004) J Physiol 561, 355-377.

3. Plagemann A, Harder T, Rake A et al. (2000) J Nutr 130, 2582-2589.

4. Plagemann A, Waas T, Harder T et al. (2000) Neuropeptides 34, 1-6.

5. Ikenasio-Thorpe BA, Breier BH, Vickers MH et al. (2007) J Endocrinol 193, 31-37.

6. Desai M, Gayle D, Babu J et al. (2005) Am J Physiol Regul Integr Comp Physiol 288, R91-R96.

7. Bellinger L, Sculley DV \& Langley-Evans SC (2006) Int J Obes 30, 729-738.

8. Vickers MH, Breier BH, Cutfield WS et al. (2000) Am J Physiol Endocrinol Metab 279, E83-E87. 